\title{
RATIONAL APPROXIMATIONS FOR THE HOLTSMARK DISTRIBUTION, ITS CUMULATIVE AND DERIVATIVE
}

\author{
D. G. HUMMER ${ }^{\dagger}$ \\ Institut für Astronomie und Astrophysik der Universität München, Scheinerstr. 1, D-8000 \\ München 80, Federal Republic of Germany
}

(Received 9 September 1985)

\begin{abstract}
The convergent series expansions of the Holtsmark distribution $P(\beta)$, its cumulative $Q(\beta)$, its derivative $R(\beta)$ and the semiconvergent asymptotic series for these functions are used to calculate rational approximations for $P, Q$, and $R$, which are valid for all positive $\beta$ and have maximum errors of approximately $10^{-8}, 10^{-9}$ and $10^{-7}$, respectively.
\end{abstract}

\section{INTRODUCTION}

Despite the great advances over the past three decades in the theory of plasma microfield distribution functions, which has been conveniently summarized by Iglesias, Hooper and DeWitt, ${ }^{1}$ the distribution function derived by Holtsmark ${ }^{2}$ is still of considerable value in dealing with certain properties of low-density, high-temperature plasmas in which particle correlations are unimportant. Recently, the need for the evaluation of the Holtsmark distribution, its cumulative and derivative has arisen in the calculation of atomic partition functions. ${ }^{3}$ The technique of Padé approximants is used to calculate rational approximations for these three functions which are valid for all values of the reduced field strength $\beta$.

\section{ANALYSIS}

We consider the Holtsmark distribution function

$$
P(\beta)=(2 \beta / \pi) \int_{0}^{\infty} \mathrm{d} t t \sin \beta t \mathrm{e}^{-t^{3 / 2}}=(2 / \beta \pi) \int_{0}^{\infty} \mathrm{d} t t \sin t \mathrm{e}^{-(t / \beta)^{3 / 2}},
$$

its cumulative

$$
Q(\beta)=\int_{0}^{\beta} \mathrm{d} \beta^{\prime} P\left(\beta^{\prime}\right)
$$

and its derivative, which we here call $R(\beta)$. Expanding $\sin \beta t$ in the first integral in Eq. (1) and integrating term by term, we obtain

$$
P(\beta)=(4 / 3 \pi) \beta^{2} \sum_{n=0}^{\infty} a_{n} \beta^{2 n}, \quad a_{n} \equiv(-1)^{n} \Gamma\left(\frac{4}{3} n+2\right) / \Gamma(2 n+2) .
$$

Integrating and differentiating this expression yields

$$
Q(\beta)=(4 / 9 \pi) \beta^{3} \sum_{n=0}^{\infty} b_{n} \beta^{2 n}, \quad b_{n} \equiv 3 a_{n} /(2 n+3),
$$

+ Staff Member, Quantum Physics Division, National Bureau of Standards. Permanent address: Joint Institute for Laboratory Astrophysics, University of Colorado and National Bureau of Standards, Boulder, CO 80309 , U.S.A. 
and

$$
R(\beta)=(8 / 3 \pi) \beta \sum_{n}^{\infty} c_{0} \beta^{2 n}, \quad c_{n} \equiv(n+1) a_{n} .
$$

The coefficients in Eqs. (3), (4) and (5) are normalized so that $a_{n}-b_{n}=c_{0}=1$; values for larger values of $n$ are readily generated by recursion.

To obtain asymptotic expressions, we follow Holtsmark ${ }^{2}$ by expanding [exp $\left.\cdots(t / \beta)^{1}\right]$ in the second form of Eq. (1), then replacing $\sin t$ by $\operatorname{Im} \mathrm{e}^{\text {it }}$. Defining a new variable of integration $u=-i$, we obtain

$$
P(\beta) \sim(2 / \pi \beta) \sum_{n=0}(1)^{n+1} \sin (3 n \pi / 4) \Gamma\left(\frac{3 n}{2}+2\right) \beta 3 n \cdot 2 / n ! .
$$

Terms with $n=0,4,8, \ldots$ vanish. It is convenient to shift the index of summation by one so that the first term is nonzero:

$$
P(\beta) \sim(15 / 8) \sqrt{2 / \pi} \beta^{-5 / 2} \sum_{m=0} \tilde{a}_{m} \beta^{\cdots \cdots}=
$$

where

$$
\tilde{a}_{m} \equiv(8 / 15) \sqrt{2 / \pi} \sin (3(m+1) \pi / 4) \Gamma\left(\frac{3 m+7}{2}\right) / \Gamma(m+2)
$$

we have chosen the normalization so that $\hat{a}_{0}=1$. Recursion relations for $\hat{a}_{m}$ are easily derived from those for $\Gamma(n)$. Integrating and recalling that $P(\beta)$ is normalized to unity, we have

$$
\begin{aligned}
& Q(\beta)=1-\int_{\beta}^{\infty} \mathrm{d} \beta^{\prime} P\left(\beta^{\prime}\right) \sim 1 \cdots(5 / 4) \sqrt{2 / \pi} \beta \cdot 2 \sum_{m_{i}=\tilde{b}_{n} \beta} \tilde{3}^{m !}, \\
& \check{b}_{m}=\check{a}_{m} / m+1 .
\end{aligned}
$$

Differentiating Eq. (7) leads immediately to

$$
R(\beta) \sim(-75 / 16) \sqrt{2 / \pi} \beta^{-7 / 2} \sum_{m=1} \tilde{c}_{m} \beta^{3 m / 2}, \quad \tilde{c}_{m}=(1+3 m / 5) \tilde{a}_{m} .
$$

\section{RATIONAL APPROXIMATIONS}

We now calculate rational approximations to $P(\beta), Q(\beta)$ and $R(\beta)$ in the following form:

$$
\begin{aligned}
& 0 \leq \beta \leq \beta^{*}: \quad P(\beta)=(4 / 3 \pi) \beta^{2} u_{1}(x) / v_{1}(x), \\
& Q(\beta)=(4 / 9 \pi) \beta^{3} u_{2}(x) / v_{2}(x), \\
& R(\beta)=(8 / 3 \pi) \beta u_{3}(x) / v_{3}(x): \\
& \beta^{*} \leq \beta<\infty: \quad P(\beta)=(15 / 8) \sqrt{2 / \pi} \beta^{-5 / 2} u_{4}(y) / v_{4}(y),
\end{aligned}
$$


Table 1. Coefficients for $P(\beta)$.

\begin{tabular}{|c|c|c|c|c|c|c|c|c|c|c|c|c|}
\hline $\mathrm{n}$ & \multicolumn{3}{|c|}{$c_{1 n}$} & \multicolumn{3}{|c|}{${ }^{d} \ln$} & \multicolumn{3}{|c|}{$c_{4 n}$} & \multicolumn{3}{|c|}{$d_{4 n}$} \\
\hline & & & & $=5.35$ & , $\mathrm{L}_{1}$ & 12 & $=13$ & ${ }_{4}=M_{4}$ & -12 & & & \\
\hline 1 & 5.3213 & 58196 & $9315-2$ & 5.1623 & 99953 & $7559-1$ & -5.8279 & 10992 & $3206+1$ & -6.3385 & 57111 & $2344+1$ \\
\hline 2 & 9.6792 & 05787 & $7047-3$ & 1.2611 & 69195 & $1988-1$ & 1.5802 & 12612 & $3226+3$ & 1.8894 & 51071 & $1591+3$ \\
\hline 3 & 4.0835 & 19154 & $2469-4$ & 1.9324 & 87786 & $9202-2$ & -2.5497 & $9130 !$ & $9232+4$ & $-3,4231$ & 19239 & $9947+4$ \\
\hline 4 & 3.1725 & 79323 & $7450-5$ & 2.0744 & 70827 & $3751-3$ & 2.6383 & 10629 & $3289+5$ & 4.1165 & 14954 & $9442+5$ \\
\hline 5 & 9.3347 & 92974 & $9771-7$ & 1.6494 & 11125 & $0278-4$ & -1.7461 & 32102 & $3968+6$ & -3.3708 & 17266 & $3750+6$ \\
\hline 6 & 4.0297 & 73892 & $3818-8$ & 1.0006 & 54492 & $167 I-5$ & 6.6626 & 75117 & $1900+6$ & 1.8369 & 81594 & $1601+7$ \\
\hline 7 & 1.2634 & 59051 & $1209-10$ & 4.6967 & 04829 & $4008-7$ & -7.3155 & 45947 & $2329+6$ & -5.9159 & 11859 & $7522+7$ \\
\hline B & 1.8177 & 22697 & $7773-11$ & 1.7082 & 31490 & $7411-8$ & -5.2626 & 44361 & $9572+7$ & 4.9132 & 01859 & $9912+7$ \\
\hline 9 & 1.5079 & 59904 & $3503-13$ & 4.7633 & 89532 & $2910-10$ & 2.4110 & 43181 & $9602+8$ & 4.5437 & 54584 & $6985+8$ \\
\hline 10 & 2.0246 & 46471 & $2406-15$ & 9.9179 & 96748 & $2997-12$ & -3.1988 & 13057 & $5441+8$ & $-2.1 \cap 12$ & 34228 & $3505+9$ \\
\hline 11 & 1.4246 & 57599 & $3322-19$ & 1.4634 & 21335 & $3989-13$ & -2.6036 & 18538 & $7058+7$ & 3.8908 & 91660 & $4974+9$ \\
\hline 12 & 1.5130 & 90778 & $2746-20$ & 1.3749 & 76403 & $5118-15$ & $-3,7993$ & 38432 & $7787+6$ & -2.7548 & 75377 & $6651+9$ \\
\hline 13 & & & & 6.2306 & 99595 & $2226-18$ & & & & & & \\
\hline
\end{tabular}

$$
\begin{aligned}
& Q(\beta)=1-(5 / 4) \sqrt{2 / \pi} \beta^{-3 / 2} u_{5}(y) / v_{5}(y), \\
& R(\beta)=(-75 / 16) \sqrt{2 / \pi} \beta^{-7 / 2} u_{6}(y) / v_{6}(y),
\end{aligned}
$$

where

$$
x \equiv \beta^{2}, \quad y \equiv \beta^{-3 / 2},
$$

and

$$
u_{i}(t) \equiv \sum_{n=0}^{L_{i}} c_{\mathrm{in}} t^{n}, \quad v_{i}(t) \equiv \sum_{n=0}^{M_{i}} d_{\mathrm{in}} t^{n}, \quad i=1,2, \ldots, 6
$$

The coefficients $c_{\text {in }}$ and $d_{\text {in }}$ are independent of $\beta^{*}$ and are equal to unity for $n=0$. The value of $\beta^{*}$ may be different for each of the three functions, and is chosen after the coefficients are evaluated so as to minimize the error.

The coefficients $c_{\text {in }}$ and $d_{\text {in }}$ are determined by equating in turn each rational expression (11)-(16) to the appropriate series from Sec. 2, cross multiplying and formally equating like powers of $x$ or $y$, as described, for example, by Baker; ${ }^{4}$ this is the simplest form of the Padé approximation procedure. The resulting linear systems are described by matrices with elements that depend only on the difference of row and column indices, i.e. so-called Toeplitz matrices. We exploit this feature of the system matrix, using a subroutine written by G. B. Rybicki, in order to reduce computing time and to increase accuracy. The calculations have been carried out with 30 sf arithmetic for a number of choices of the polynomial orders $L_{i}$ and $M_{i}$.

To select the optimum values of $L_{i}, M_{i}$ and $\beta^{*}$, accurate values of $P(\beta)$ and $R(\beta)$ were evaluated by direct numerical integration using the form

$$
P(\beta)=(2 / \beta \pi) \int_{0}^{2 \pi} \mathrm{d} t \sin t \sum_{n=0}(2 n \pi+t) \exp \left\{-[(2 n \pi+t) / \beta]^{3 / 2}\right\},
$$


Table 2. Coefficients for $Q(\beta)$.

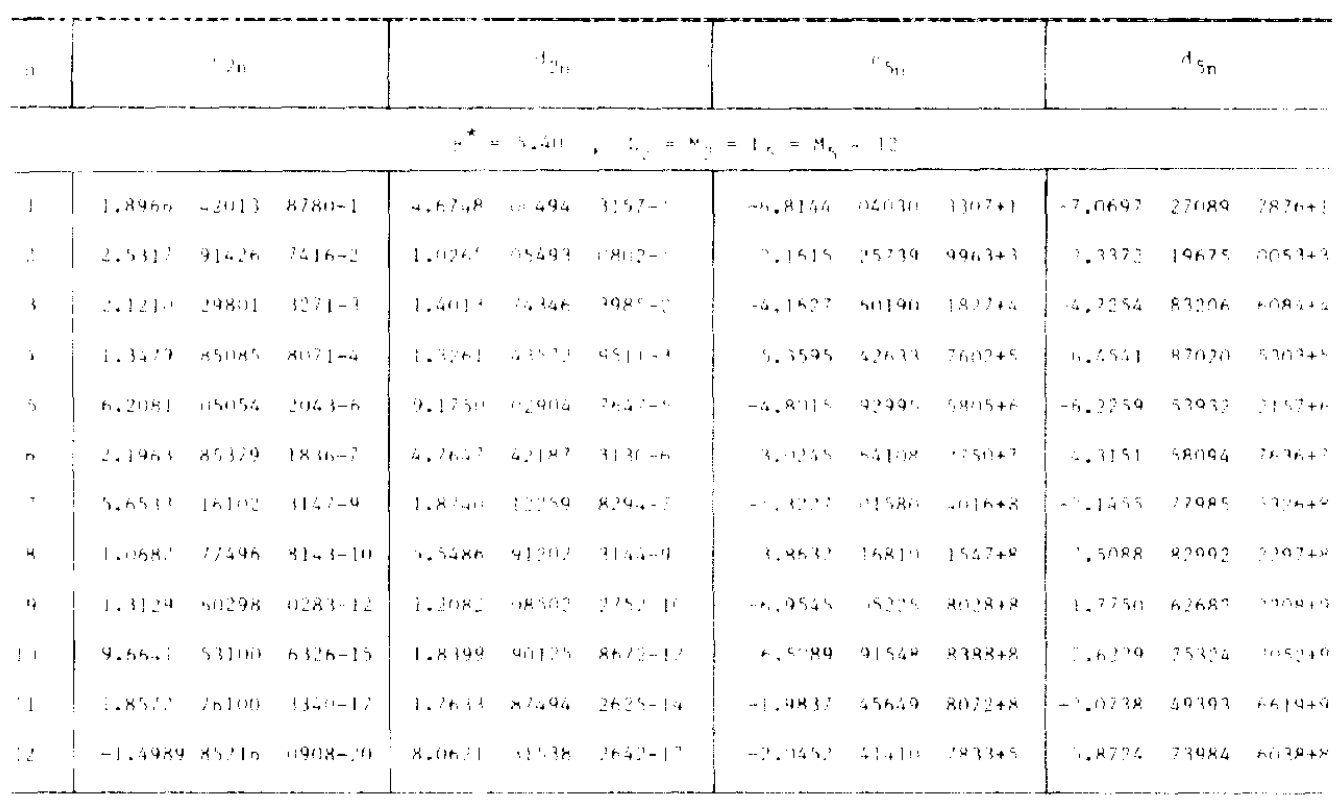

together with a similar expression for $R(\beta)$. Error curves were computed for each rational approximation for the interval $4.0 \leq \beta \leq 6.0$, in which the small- $\beta$ and large- $\beta$ forms were expected to have comparable errors. By choosing the small- $\beta$ and large- $\beta$ form with the smallest errors, plotting the error curves and locating the intersection point, we determined for $P(\beta)$ the optimum value $\beta^{*}=5.35$, which corresponds to a maximum relative error $\epsilon_{\max }=8.9 \times 10^{9}$ for the approximations with $L_{1}=12, M_{1}=13, L_{4}=M_{4}=$ 12. For $R(\beta)$, we find $\beta^{*}=5.15, \epsilon_{\max }=1.6 \times 10^{\prime}$ for $L_{3}=13, M_{3}=14, L_{6}=$ $14, M_{\mathrm{b}}-13$. No check values were computed for $Q(\beta)$, but by comparing the small $-\beta$ and large $-\beta$ forms for $L_{2}=M_{2}=L_{5}=M_{5}=12$ in the neighborhood of $\beta=5.0$, we find $\beta^{*}=5.40$ and $\epsilon_{\max } \simeq 1 \times 10^{-9}$. The coefficients of the rational approximations

Table 3. Coefficients for $R(\beta)$.

\begin{tabular}{|c|c|c|c|c|c|c|c|c|c|c|c|c|}
\hline$n$ & & ${ }^{\prime}$ in & & & 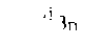 & & & $\mathrm{b}$, & & \multicolumn{3}{|c|}{1 in } \\
\hline & & & $B^{*}=$ & 5 & $13=13$ & . $\quad M_{3}=$ & - $\mathrm{I}_{h}=$ & $\therefore \quad$ & $a_{h}=11$ & & & \\
\hline i & -3.7714 & $: 3754$ & $2388-1$ & $5.489: 1$ & 74511 & $88\{7-1$ & -7.6330 & 6.5660 & $3994+1$ & $+9.45 m$ & 40459 & hallot: \\
\hline$\therefore$ & $2.8+58$ & 82706 & $1720-3$ & 1.4339 & $A\left(1 f_{0}\right) \mathrm{i}$ & A $754-1$ & 2.7570 & 50239 & $f+x) 1+7$ & $\therefore, \therefore 15$ & $894: 7$ & $8 Q ? \mathrm{C}_{3}+3$ \\
\hline$i$ & -2.4970 & 47597 & $8578-3$ & 2.3654 & 23418 & $8604-3$ & -6.1205 & 28191 & $0748+4$ & $-\hat{x},+429$ & 65578 & $322+$ \\
\hline 4 & -1.0908 & 00305 & $5922-5$ & 2.7557 & 98165 & $8846-3$ & 9.1767 & 67955 & $5383+5$ & 1.5163 & $54 ? 14$ & $2104+6$ \\
\hline 5 & -5.9411 & 20278 & $1125-\theta$ & 2.4012 & 66396 & 8210.4 & -9.5479 & 93996 & Inkath & $1.97+9$ & 29297 & $4955+=$ \\
\hline 6 & -3.0835 & 58842 & $5598-8$ & 1.6159 & 55789 & $5932-5$ & 7.1555 & 23640 & $0707+?$ & $1.944 ?$ & 22104 & $1642+8$ \\
\hline 7 & -6.0080 & 850145 & $0585-9$ & 8.3441 & 37592 & $27016-1$ & -3.6602 & 83959 & $2271+8$ & -1.3194 & $5.845 \mathrm{~h}$ & $95 h a+c$ \\
\hline 8 & -1.3513 & 40679 & $4647-11$ & 3.5723 & 07268 & $9829-k$ & 1.2099 & 42069 & $11420+9$ & 7.0598 & 13144 & $14+4+4$ \\
\hline 9 & -2.4485 & 98156 & $7752-12$ & 1.1773 & 49943 & $0426-9$ & -2.1701 & 95814 & $817 n+9$ & -2.7760 & $3043 ?$ & $?+1 ?+1$ \\
\hline 10 & 4.5538 & 91752 & $5173-15$ & $3.016 ?$ & 15665 & $4715-11$ & 7.8558 & 28079 & $8258+8$ & 7.7500 & 6,6977 & $2928+1$ \\
\hline 11 & -3.2230 & 60591 & $6261-16$ & 5.8399 & 29379 & $7887-13$ & 2.5883 & 18206 & $9229+9$ & -1.4429 & 55383 & $2897+!$ \\
\hline 12 & 1.5088 & 21629 & $8286-18$ & 8.0938 & $9346 \mathrm{~h}$ & $2632-15$ & -8.2367 & 64554 & $9789+7$ & 1.5867 & 62018 & $3909+11$ \\
\hline 13 & -7.4026 & 17976 & $4|04-2|$ & 7.2087 & 55477 & $9050-17$ & -1.0598 & 79446 & $6865+8$ & -7.6147 & (19R94 & $7+5 ?+10$ \\
\hline 14 & & & & 3.1227 & $322 B 6$ & $7957-19$ & -3.8444 & 64866 & $9|R\rangle+?$ & & & \\
\hline
\end{tabular}


Table 4. Values of Functions.

\begin{tabular}{c|cc|c|c}
\hline & $P(B)$ & $O(B)$ & $R(B)$ \\
\hline 1.0 & $2.712208070-1$ & $1.086077296-1$ & $7.077383690-1$ \\
2.0 & $3.369387827-1$ & $4.517618482-1$ & $-1.374016902-1$ \\
3.0 & $1.760629272-1$ & $7.077478512-1$ & $-1.394870538-1$ \\
4.0 & $8.067354136-2$ & $8.294655532-1$ & $-5.963921261-2$ \\
5.0 & $4.118023725-2$ & $8.875444652-1$ & $-2.494381058-2$ \\
6.0 & $2.382208458-2$ & $9.189659950-1$ & $-1.177855295-2$ \\
7.0 & $1.516457557-2$ & $9.380057335-1$ & $-6.266227225-3$ \\
8.0 & $1.034976409-2$ & $9.505478667-1$ & $-3.661034529-3$ \\
9.0 & $7.438307487-3$ & $9.593291180-1$ & $-2.297705775-7$ \\
10.0 & $5.561346283-3$ & $9.657648611-1$ & $-1.524296364-3$ \\
\hline
\end{tabular}

defined by Eqs. (11)-(16) are given in Tables 1,2 and 3 for $P(\beta), Q(\beta)$ and $R(\beta)$, respectively. To enable users to check that these coefficients are correctly entered into their programs, we give in Table 4 ten-digit values of the functions for $\beta=1(1) 10$ generated by the approximations given here; not all of the digits are necessarily significant.

Acknowledgments - I am grateful to the Alexander von Humboldt-Stiftung for a Senior U.S. Scientist Award and to R. P. Kudritzki for the hospitality of his Institute.

\section{REFERENCES}

1. C. A. Iglesias, C. F. Hooper, Jr. and H. E. DeWitt, DeWitt, Phys. Rev, A 28, 361 (1983).

2. J. Holtsmark, Ann. Phys. 58, 577 (1919).

3. D. G. Hummer and D. Mihalas, Astrophys. $J$. In press.

4. G. A. Baker, Jr., Essentials of Padé Approximants, p. 5. Academic Press, New York (1975). 